\title{
O papel do Neuropsicólogo na reabilitação de crianças com Transtorno do Espectro
}

\section{Autista}

The Role of the Neuropsychologist in the rehabilitation of children with Autistic Spectrum Disorder

El Papel del Neuropsicólogo en la rehabilitación de niños con Trastorno del Espectro Autista

Recebido: 10/06/2021 | Revisado: 19/06/2021 | Aceito: 24/06/2021 | Publicado: 10/07/2021

Caio Pereira de Sousa

ORCID: https://orcid.org/0000-0002-8952-0218 Centro Universitário Maurício de Nassau, Brasil

E-mail: sousacaio0596@gmail.com

Francisca Giovana Andrade de Araújo ORCID: https://orcid.org/0000-0002-6044-3896 Centro Universitário Maurício de Nassau, Brasil

E-mail: franciscagiovana@gmail.com

Valdelice Juliane Alves Caribé ORCID: https://orcid.org/0000-0001-8349-8075 Centro Universitário Maurício de Nassau, Brasil

E-mail: valdelice.juliane@gmail.com

Pedro Wilson Ramos da Conceição ORCID: https://orcid.org/0000-0002-3868-4917 Pontifícia Universidade Católica de Minas Gerais, Brasil

E-mail: Pedro_wilson_ramos@hotmail.com

Lilian Railine Ferry e Silva

ORCID: https://orcid.org/0000-0002-6920-9422 Centro Universitário Maurício de Nassau, Brasil

E-mail: lilianferryy@gmail.com

Sâmia Karina Lima Soares

ORCID: https://orcid.org/0000-0002-0663-259X Centro Universitário Maurício de Nassau, Brasil E-mail: samiakaryna@hotmail.com

Jardell Saldanha Amorim

ORCID: https://orcid.org/0000-0002-4301-4088 Centro Universitário Maurício de Nassau, Brasil E-mail: jardellamorim@yahoo.com.br Jacqueline da Silva Santos ORCID: https://orcid.og/0000-0001-9240-3867 Centro Universitário Maurício de Nassau, Brasil E-mail: Jack.10silva@hotmail.com

Sabrina Amorim Paulo

ORCID: https://orcid.org/0000-0002-9339-0612 Centro Universitário Maurício de Nassau, Brasil E-mail: samorimpaulo@gmail.com

Regina Lucia dos Reis e Silva ORCID: https://orcid.org/0000-0001-5850-2300 Centro Universitário Maurício de Nassau, Brasil E-mail: reginallrreis@gmail.com

Ulaidia Betânia da Silva Sousa ORCID: https://orcid.org/0000-0003-2819-4102 Centro Universitário Maurício de Nassau, Brasil E-mail: ulaidiabss@gmail.com

Ana Virgínia Nunes Soares ORCID: https://orcid.org/0000-0001-5339-8655 Centro Universitário Maurício de Nassau, Brasil E-mail: avnsoares@hotmail.com

Maria Michele de Resende Sousa ORCID: https://orcid.org/0000-0002-2742-5231 Centro Universitário Maurício de Nassau, Brasil

E-mail: mariamicheleresende@gmail.com

Karoline Marques da Silva Freitas ORCID: https://orcid.org/0000-0002-6359-6062 Centro Universitário Maurício de Nassau, Brasil

E-mail: karolinesfreitas@outlook.com.br 


\title{
Resumo
}

A neuropsicologia é uma área de atuação consideravelmente nova perante a normalização da psicologia no Brasil em seus 59 anos. Posto que, a prática do neuropsicólogo vem se mostrando primordial junto a equipe multi e interdisciplinar no processo de avaliação e reabilitação das crianças com transtorno do espectro autista. Esse estudo irá apresentar a atuação do neuropsicólogo no atendimento e reabilitação de crianças com TEA. Trata-se de uma entrevista qualitativa semi-estruturada realizada com duas neuropsicólogas, utilizando a análise de conteúdo de Bardin para descrição dos resultados. Observou-se que o neuropsicólogo auxilia nas avaliações inicias, além de fornecer assistências para equipe e familiares sobre o manejo que deve-se adotar na reabilitação do paciente, ainda que, encontram-se dificuldades, na prática em equipe, contudo, a atuação em grupo é necessária para um diagnóstico completo e reabilitação. Portanto, o neuropsicólogo tornou-se um profissional presente nas equipes de reabilitação de crianças com TEA, canto nos hospitais como nos centros especializados, tendo em vista que, com seus conhecimentos o processo de desenvolvimento ganhar qualidade e praticidade no decorrer do tratamento.

Palavras-chave: Neuropsicologia; Reabilitação; Transtorno do espectro autista.

\begin{abstract}
Neuropsychology is a considerably new field of activity in face of the normalization of psychology in Brazil in its 59 years. Since, the practice of neuropsychologist has been shown to be essential with the multi and interdisciplinary team in the process of assessment and rehabilitation of children with autism spectrum disorder. This study will present the role of the neuropsychologist in the care and rehabilitation of children with ASD. This is a semi-structured qualitative interview conducted with two neuropsychologists, using Bardin's content analysis to describe the results. It was observed that the neuropsychologist helps in the initial stories, in addition to providing assistance to the team and family members on the management that should be adopted in the patient's rehabilitation, although difficulties are specified in team practice, however, acting in group is required for full diagnosis and rehabilitation. Therefore, the neuropsychologist has become a professional present in the rehabilitation teams of children with ASD, singing in hospitals as well as in specialized centers, considering that, with their knowledge, the development process gains quality and practicality during the treatment.
\end{abstract}

Keywords: Neuropsychology; Rehabilitation; Autistic spectrum disorder.

\begin{abstract}
Resumen
La neuropsicología es un campo de actividad considerablemente nuevo frente a la normalización de la psicología en Brasil en sus 59 años. Desde entonces, la práctica del neuropsicólogo ha demostrado ser fundamental con el equipo multidisciplinario e interdisciplinario en el proceso de evaluación y rehabilitación de niños con trastorno del espectro autista. Este estudio presentará el papel del neuropsicólogo en el cuidado y rehabilitación de niños con TEA. Esta es una entrevista cualitativa semiestructurada realizada con dos neuropsicólogos, utilizando el análisis de contenido de Bardin para describir los resultados. Se observó que el neuropsicólogo ayuda en los relatos iniciales, además de brindar asistencia al equipo y familiares en el manejo que se debe adoptar en la rehabilitación del paciente, aunque se especifican dificultades en la práctica en equipo, sin embargo, se requiere actuar en grupo. para un diagnóstico completo y rehabilitación. Por ello, el neuropsicólogo se ha convertido en un profesional presente en los equipos de rehabilitación de niños con TEA, cantando tanto en hospitales como en centros especializados, considerando que, con sus conocimientos, el proceso de desarrollo gana calidad y practicidad durante el tratamiento.
\end{abstract}

Palabras clave: Neuropsicología; Rehabilitación; Trastorno del espectro autista.

\section{Introdução}

Nesse artigo será apresentado o papel do neuropsicólogo no processo de reabilitação de crianças com transtorno do espectro autista (TEA) e as intervenções que o psicólogo utiliza a fim de contribuir para o desenvolvimento dessas crianças no meio social e contexto escolar.

De acordo com o Manual de diagnóstico e Estatístico de Transtornos Mentais (DSM-V, 2013), o transtorno do espectro autista, está classificado dentro dos transtornos do neurodesenvolvimento, apresentando características como: Déficit persistente na comunicação social e na interação social em múltiplos contextos, a gravidade baseia-se em prejuízos na comunicação e em padrões restritos ou repetitivos de comportamento.

A neuropsicologia ainda é considerada nova no meio acadêmico, e mediante a temática abordada nesse estudo, é de 
suma importância trazer pesquisas sobre o seu desenvolvimento e as novas hipóteses que ela vem a desenvolver para a sociedade como um todo (Fuentes, 2014). Diante dessa condição objetiva da realidade, surgiu o seguinte questionamento: como o neuropsicólogo atua no processo de reabilitação e desenvolvimento de crianças com TEA?

A temática abordada contribuirá tanto para os pesquisadores quanto para a sociedade de forma geral, fazendo com que, sejam agregados conhecimentos acerca do diagnóstico do TEA, tendo em vista que, o espectro é identificado através da observação comportamental da criança e por entrevistas com os cuidadores. Além disso, é importante ressaltar que, nas últimas décadas, o número de crianças, diagnosticadas com autismo tem crescido de uma forma significativa em todo o mundo (Griesi-Oliveira \& Sertíe, 2017).

A presente pesquisa se faz importante, pois, tem o objetivo de apresentar a atuação do neuropsicólogo no atendimento e reabilitação de crianças com TEA. É observado que muitas vezes a primeira avaliação é realizada no hospital, e logo é encaminhado para um centro de reabilitação especializado, aonde possui uma estrutura adequada para fornecer os cuidados necessários.

\section{Metodologia}

A pesquisa é destinada aos acadêmicos e profissionais da saúde, fornecendo conhecimentos científicos a respeito da contribuição do neuropsicólogo na reabilitação de crianças autistas. O estudo teve sua elaboração inicialmente através de uma análise bibliográfica acerca da atuação do neuropsicólogo, das avaliações realizadas e dos processos de intervenções utilizados no desenvolvimento do paciente com TEA. Em seguida foi realizada uma pesquisa de campo de caráter qualitativo. A entrevista qualitativa tem como base a ênfase no estudo de microprocessos, a partir das análises de ações sociais, sendo estas individuais ou grupais, assim são ressaltadas principalmente as experiências e a subjetividade de cada indivíduo. (Patias \& Hohendorff, 2019). Segundo Yin (2010) as perguntas em uma entrevista devem ser abertas, pois de acordo com o contexto inserido pode-se fazer alterações para não limitar as respostas dos entrevistados.

Foram realizadas entrevistas semiestruturadas com duas neuropsicólogas que atuam em um centro de reabilitação especializado e em um hospital infantil, considerando que as profissionais possuem experiências práticas com o processo de reabilitação de crianças com TEA. Sendo as mesmas devidamente informadas a respeito do estudo e seus direitos na pesquisa de acordo com a resolução 466/12 do Ministério da Saúde (Brasil, 1996) e sobre a resolução n 510/2016 acerca da ética em pesquisas em ciências humanas e sociais. A entrevista semiestruturada é uma ferramenta importante no processo de interação social, no qual o entrevistador tem como objetivo obter informações do entrevistado através de um roteiro de entrevista (Batista, Matos \& Nascimento, 2017). Para preservar a ética e dignidade do estudo e dos entrevistados, foi fornecido o termo de consentimento de livre esclarecimento (TCLE), que consiste em informar ao sujeito a respeito do seu envolvimento na pesquisa, para que então possa decidir com autonomia e responsabilidade sobre a sua participação. As entrevistadas assinaram o TCLE, entregue em duas vias, uma sendo fornecida para o participante e outra ficando com o pesquisador.

Todas as entrevistas foram gravadas com o auxílio de um gravador de áudio (celular) e ao final, as mesmas foram transcritas e analisadas de acordo com o método de análise de conteúdo. Segundo Bardin (2011) a análise de conteúdo consiste em três etapas, a pré-analise, exploração do material e tratamento dos resultados, a inferência e a interpretação. A pré-análise foi realizada a partir das transcrições feitas nas entrevistas para organização dos objetivos do corpos da pesquisa; Na fase de exploração do material, foram escolhidas as unidades de codificação, sendo feita uma seleção de regras, escolha das categorias, enumeração, classificação e agregação; Na fase de tratamento dos resultados, realizou-se a inferência e a interpretação dos dados obtidos, assim validando as informações coletadas nas entrevistas, sendo apresentadas no tópico de resultados e discussão. A análise de conteúdo, segundo a perspectiva de Mozzato \&e Grzybovski (2011) consiste em uma técnica 
metodológica podendo-se aplicar em diversos discursos e a todas as formas de comunicação, seja qual for à natureza do seu suporte.

\section{Resultados e Discussão}

\subsection{Avaliação neuropsicológica do autismo}

A avaliação neuropsicológica é um instrumento que tem como objetivo avaliar as funções executivas, deste modo, auxilia no desenvolvimento e formulação de estratégias para alcançar os objetivos. Estes que são fornecidos através do resultado da avaliação, com o intuito de trabalhar os aspectos cognitivos, emocionais e comportamentais (Blakleitz, Paris \& Souza, 2017). Como podemos observar através das entrevistas que o método de avaliação neuropsicológica é realizado no primeiro encontro com o paciente, utilizando-se a observação comportamental com as crianças e perguntas estruturadas com os cuidadores.

[...] O primeiro ponto, observamos o desenvolvimento da criança, que aspectos do desenvolvimento não estão dentro do esperado para aquela idade, se existem atrasos ou alterações em alguma função importante que levariam a suspeita do transtorno do espectro autista. A partir dessa compreensão do desenvolvimento do paciente, existe uma hipótese mais ou menos segura de que a criança tem o diagnóstico de autismo [...]. (Psicóloga A, 2020).

[...] No centro fazemos à avaliação inicial, chamada de avaliação global, dentro dessa avaliação observamos todos os aspectos da criança em relação à cognição, comportamento, emoções, comunicação e aspecto da linguagem [...]. (Psicóloga B, 2020).

Podemos observar mediante a fala das entrevistadas que o método inicial da avaliação neuropsicológica é a observação, é através dela, que o psicólogo vai formular suas primeiras hipóteses a respeito do diagnóstico. Para Neumann et al. (2017), a avaliação é um instrumento fundamental para o diagnóstico do autismo, tendo em vista que, para uma identificação complexa como a do TEA, ainda seja confirmado exclusivamente por meio da observação clínica.

No centro de reabilitação, após a criança com o transtorno intelectual passar pela avaliação global, esta é enquadrada dentro de um grupo específico para que possa dar início ao trabalho de reabilitação. A “psicóloga B' também relata que, quando há uma suspeita de que a criança não tem um transtorno intelectual e sim alguma outra dificuldade no aprendizado, ela é encaminhada para uma avaliação neuropsicológica mais detalhada para que então, o diagnóstico dessa criança possa ser fechado.

[...] Quando realizamos uma avaliação, enquadramos o paciente em algum procedimento dentro do centro para poder iniciar o tratamento, entretanto, para que a criança possa ser enquadrada, ela precisa ter uma deficiência intelectual (Síndrome de Down ou Autismo), quando o psicólogo observa que existe algo a mais ou algo a menos, por exemplo: quando não está dentro do espectro, mas pode ter um possível TDAH ou outra comorbidade associada com transtornos mentais, então encaminhamos para uma avaliação neuropsicológica, e a partir desta, o profissional vai avaliar se ele tem o perfil ou não para ser enquadrado nos grupos, isso é uma situação atípica [...]'”. (Psicóloga B, 2020).

Segundo Silva e Milick (2009), uma avaliação neuropsicológica colabora para a comprovação e também serve para descartar suspeitas. Após a confirmação ou não do diagnóstico de TEA a equipe determina quais procedimentos irão abordar com aquela criança, e se ela vai continuar no grupo de reabilitação ou irá ser encaminhada para outros profissionais.

Outro método de avaliação realizado no centro de reabilitação mencionado pela 'Psicóloga B' é a avaliação de linha de base, elaborado no segundo encontro do psicólogo com a paciente, nele o profissional vai fazer uma observação mais ampla das diversas áreas do desenvolvimento e dos déficits apresentados e então, planejará as intervenções adequadas para a 
demanda de cada grupo de crianças nos próximos seis (6) meses:

[...] No segundo encontro é realizado uma avaliação de linha de base, onde o psicólogo vai analisar avaliar e observar as áreas da cognição, comportamento e compreensão de comando. Nessa avaliação observam-se como está o contato visual dessa criança, o processo imaginativo, a interação inicial, a atenção compartilhada, a compreensão de comandos e a execução de habilidades de comunicação, possíveis dificuldades sensoriais e a parte de coordenação motora ampla e fina. Todos os requisitos para que seja planejada as intervenções nos próximos 6 meses [...]. (Psicóloga B, 2020).

Benitez et al. (2020) enfatiza a importância de uma avaliação sistematizada para um apanhado dos aspectos cognitivos e comportamentais, com o objetivo de obter um repertório inicial (linha de base), para cada aspecto identificado. Além dos métodos mencionados pelas entrevistas, Silva e Mulink (2009) mencionam que a Childhood Autism Rating Scale - CARS (Escala de Avaliação de Autismo na Infância) é utilizada para avaliar o comportamento da criança de acordo com a entrevista realizada com os pais.

Vilar (2019) afirma que nos dias atuais a avaliação neuropsicológica contribui significativamente nos mais diversos contextos psicológicos, fornecendo importantes colaborações para o entendimento do funcionamento cerebral e os processos cognitivos onde o neuropsicólogo tem a função de relacionar as observações do comportamento do paciente com os critérios para fechar o diagnóstico, e quanto mais rápidos o paciente for diagnosticado, melhor será o prognóstico para a reabilitação.

\subsection{Técnicas que auxiliam na reabilitação do desenvolvimento autista}

Com relação às técnicas utilizadas pelas neuropsicólogas que atuam com pacientes com TEA, ambas confirmaram que o método Applied Behavior Analysis (ABA) que é desenvolvido dentro da Análise do Comportamento (AC), e comprovado cientificamente como a metodologia com mais resultados no desenvolvimento cognitivo de crianças autistas.

[...] Basicamente as alterações são feitas dentro do método ABA, que é o método mais utilizado na análise do comportamento, que são as estratégias comportamentais onde você vai treinar aquela paciente para desenvolver as habilidades que estão alteradas por conta do transtorno, a criança ou adulto vai ser habilitada a desenvolver determinadas habilidades que ela não possui ou não possui elevadamente [...]. (Psicóloga A, 2020).

[...] Aqui utilizamos como embasamento teórico, o neuropsicólogo, à análise aplicada do comportamento, o ABA, assim, a gente trabalha nessa linha porque cientificamente falando o ABA, é que tem realmente comprovação de eficácia [...]. (Psicóloga B, 2020).

Setúbal (2018) traz que, de acordo com Associação para a Ciência do Tratamento do Autismo dos Estados Unidos, o método ABA é comprovado cientificamente como um tratamento eficaz para a reabilitação autista. O método realiza um intensivo ensino, individualizando as habilidades necessárias que a criança necessita para adquirir um melhor desenvolvimento na qualidade de vida.

Desse modo, a família se torna um fator importante para que a criança desenvolva as suas habilidades, bem como o local que os psicólogos estão trabalhando com elas. Para isso os profissionais vão utilizar a psicoedução com a família, para orientar no manuseio da criança. Ao que é relacionado às práticas fora do hospital ou do centro de reabilitação, as psicólogas afirmam:

[...] A intervenção vai partir do perfil de cada paciente, de modo geral é voltada inicialmente para orientação da família, porque, sabemos que o autismo causa déficits em algumas áreas no desenvolvimento da criança, principalmente nas esferas de relações interpessoais. Inicialmente a família é psicoeducada acerca do transtorno para que ela consiga entender as alterações que aquele paciente apresenta, e que tipo de intervenções vão ser feitas e com quais objetivos [...]. (Psicóloga A, 2020). 
[...] É orientado como eles devem fazer caso o manejo seja de uma maneira mais simples, não técnico, quando a família não tem as técnicas, mas, é orientada de uma forma que eles possam ter uma prática adequada. Damos um tempo para essa família, geralmente eu peço o retorno de orientação em três meses, a família volta e me diz o que conseguiu e o que não conseguiu [...]. (Psicóloga B, 2020).

A psicoeducação familiar é utilizada especialmente para trabalhar o entendimento da família em relação à patologia que aquele paciente apresenta, dessa forma, recorre às estratégias cognitivo-comportamental, e se beneficiam das técnicas de orientações como estratégias para aquela família (Silva, Araújo \& Maia 2018).

A psicóloga "B" discorreu sobre as técnicas e instrumentos utilizados no processo de reabilitação da criança autista. É mencionado que no centro de reabilitação os pacientes são divididos em grupos, e esses grupos são divididos de acordo com as faixas etárias, grupos de primeiro bloco é chamado de estimulação precoce, que vai de zero (0) a cinco (5) anos de idade e estimulação integrativa que é dos 5 a dezessete (17) anos. Após as crianças serem inseridas em um grupo é utilizado o instrumento de vilenand, que são perguntas a respeito do desenvolvimento do paciente autista, no qual o instrumento é aplicado nos seus respectivos responsáveis, onde são feitas perguntas estruturadas, de como ele observa o comportamento da criança. Outras metodologias utilizadas no centro de reabilitação é a agenda de rotina, no qual é ensinada pelo psicólogo uma rotina que a criança deve seguir, partindo do princípio do desenvolvimento e da organização. Também é citado o "teacher", que não é uma abordagem, mas é muito utilizado no centro de reabilitação para trabalhar a reorganização do paciente.

[...] Primeiro é utilizado um instrumento chamado vineland. Nesse instrumento são utilizadas perguntas estruturadas e diretivas ao cuidador da criança, dentro desse instrumento são apresentadas perguntas voltadas para a área da comunicação, habilidades de vida diária, atividades de coordenação motora e a área que se determina mais para o psicólogo, que é a questão Comportamental [...]. (Psicóloga B, 2020).

[...] Análise aplicada eu utilizo muito o "teacher", que é um programa psicoeducacional, não é uma abordagem, é uma metodologia que a gente emprega também para trabalhar a organização do paciente, que é muito utilizado em neuropsicopedagogia, é uma forma que a gente trabalha muito a reorganização de pacientes em atendimento educacional especializado, e dentro da minha atuação eu utilizo muito como questão de agenda de rotina [...]. (Psicóloga B, 2020).

Segundo Almeida, Boueri e Postallia (2016) a escala de vineland é reconhecida globalmente por sua eficácia na avaliação dos comportamentos adaptativos da criança com TEA, a escala serve como uma associação ao teste de inteligência que servirá como suporte para diagnosticar se o sujeito tem uma deficiência intelectual. No que diz respeito à formulação de uma agenda de rotina para as crianças com TEA. Vila (2016), afirma que as crianças autistas modificam seu comportamento diariamente quando algo é alterado em sua rotina, por isso é necessário a criação de uma agenda de rotina para eles, com o objetivo de desenvolver uma segurança no seu dia a dia.

\subsection{Atuação do neuropsicólogo junto a equipe multidisciplinar e familiar}

Ao analisar a fala das entrevistadas sobre a atuação do neuropsicólogo junto a uma equipe multidisciplinar e interdisciplinar, é notória a importância do trabalho em conjunto com os outros profissionais, na formulação do diagnóstico e prognóstico que o paciente irá receber para sua reabilitação. A psicóloga "A" relata que no hospital infantil, para o diagnóstico ser fechado, é preciso que " A criança seja encaminhada para o neuropediatra e outros profissionais da equipe para que esse diagnóstico seja definido" (Psicóloga A), bem como a "Psicóloga B" afirma que o neuropsicólogo não atua de forma individualizada, ele sempre trabalha em equipe no centro de reabilitação, como pode-se observar na fala a seguir: 
[...] Porque trabalhamos em equipe, e o psicólogo não tem a atuação dele individualizada, mas de forma multidisciplinar e interdisciplinar, então dentro da nossa equipe, nós temos o fonoaudiólogo, terapeuta ocupacional e psicopedagogo [...]. (Psicóloga B, 2020).

A equipe multidisciplinar tem como objetivo analisar cada elemento de forma individual, pois, cada profissional executa o parecer específico de sua especialidade. Já a equipe interdisciplinar tem o seu trabalho envolvendo todos os profissionais funcionando de maneira uniforme e colaborativa, ou seja, os integrantes da equipe interagem entre si em busca de uma melhor qualidade de vida para os pacientes (Zanchett \& Dallacosta, 2015).

No que diz a respeito ao trabalho multidisciplinar e interdisciplinar, todos que acompanham esse processo de reabilitação, são considerados a base da reabilitação dos pacientes com autismo, e sem esse suporte profissional haveria uma maior dificuldade para alcançar as metas estabelecidas para um desenvolvimento mais completo (Fialho, 2015). O trabalho em equipe vem se destacando como um recurso importante, com a intenção de remover o estigma de que é centrado na figura do médico, assim o trabalho em equipe promove uma integração em diversas áreas da saúde (Nascimento, 2020).

No que tange a fala da psicóloga "B", sobre a atuação na equipe multidisciplinar e interdisciplinar no centro de reabilitação, é relatado dificuldades, estas devido ao trabalho com vários profissionais de áreas distintas e cada um possuírem sua própria opinião para a tomada de decisões acerca dos métodos de intervenções, apesar das dificuldades, o trabalho em equipe tem muito a acrescentar para todos os profissionais, assim pode-se notar na fala a seguir:

[...] A nossa equipe é multi e interdisciplinar, portanto, nossos grupos funcionam com quatro (4) crianças ou adolescentes, porque é dividido por faixa etária, e três (3) profissionais (psicólogo fonoaudiólogo e terapeuta ocupacional). Sabemos que trabalhar em equipe é muito difícil, porque são três profissionais de áreas distintas, mas ao mesmo tempo é muito rico, porque as profissões atuam de forma diferenciada, elas se misturam ao mesmo tempo em que uma complementa a outra [...]. (Psicóloga B, 2020).

Para Silva e Ruiz (2018), as dificuldades encontradas dentro de uma equipe multidisciplinar mesmo com instrumentos adequados para o atendimento, é a inexperiência dos profissionais em saber manejarem as crianças no dia a dia, pois é uma área que necessita de capacitação devido à complexidade do transtorno. Entretanto, de acordo com as diretrizes do Sistema Único de Saúde (SUS), compete ao SUS promover estratégias e orientar os familiares que receberem o diagnóstico de TEA, encaminhando para locais especializados, onde profissionais habilitados irão auxiliar os cuidadores para melhor manejo dessas crianças. (Ministério da Saúde, 2013).

A psicóloga 'B', enfatizou a atuação do neuropsicólogo junto à família e cuidador da criança com TEA, ela também cita uma tríade " família, escola e terapeuta' 'é mencionado que o psicólogo que atua no centro realiza uma orientação escolar, na qual o psicólogo vai orientar os professores sobre o manejo e intervenções que ele pode desenvolver dentro da sala de aula.

[...] Nós temos um procedimento chamado de orientação escolar e nele participo, eu como psicóloga e uma psicopedagoga, então a gente faz essas orientações sobre o manejo comportamental dessa criança, de intervenções de como o professor pode atuar dentro de sala de aula, e como a família pode atuar em ambiente domiciliar, sendo uma das nossas preocupações como psicólogo [..]. (Psicóloga B, 2020).

Segundo Camargo et al. (2020), fica evidente a falta de conhecimento e preparo das escolas e professores para receberem alunos com TEA, por falta de estratégias e técnicas pré-estabelecidas dificultando assim o desenvolvimento no âmbito escolar. Dessa forma, ressalta-se a importância do neuropsicólogo, para ajudar a desenvolver as habilidades cognitivas e sociais, fornecendo uma melhor qualidade de vida para a criança. 


\subsection{Dificuldades da reabilitação neuropsicológica}

Para a psicóloga "A”, "as dificuldades são várias, uma das mais difíceis é a idade com que a criança chega para avaliação e a negligência em relação aos sintomas", outro fator a ser considerado são os longos períodos de espera "seis meses para conseguir uma consulta e quando consegue tem que voltar para próxima que é mais um ano, prejudicando o processo de reabilitação". Psicóloga A.

Segundo Pereira et al. (2021) o diagnóstico tardio interfere nas implementações das intervenções precoces dificultando o prognóstico do paciente, além de causar prejuízos significativos nas habilidades sociais, nos aspectos cognitivos e em todo o desenvolvimento. Outrossim, atrasando todo o processo de reabilitação dessas crianças.

A maior dificuldade apresentada na fala da psicóloga "A" que atua no hospital infantil é a falta da comunicação entre a equipe, o que atrapalha bastante no desenvolvimento da reabilitação da criança autista, como podemos observar na fala a seguir:

[...] NA rede pública ainda temos muito o que melhorar pois existem muitas dificuldades em relação a comunicação ao trabalho Interdisciplinar, que muitas vezes acaba sendo desfragmentado [...] (Psicóloga A, 2020).

No que tange as dificuldades em manter uma boa comunicação em equipe, a diferença de hierarquia e conflitos no trabalho influenciam diretamente na maneira que a comunicação é estabelecida, fazendo com que os profissionais tenham sua atuação desfragmentada e prejudicando o trabalho em equipe (Bagnasco et al., 2013).

$\mathrm{Na}$ fala das psicólogas é relatada uma dificuldade muito importante que deve ser observada em relação à família da criança, sobre sua questão sociocultural, se a família tem uma situação financeira e recursos para que ela possa junto ao psicólogo desenvolver uma reabilitação adequada com a criança.

[...]Uma dificuldade é a aceitação do diagnóstico por parte da família, em que muitas vezes os cuidadores chegam e sabe que a criança tem autismo, mas ela não aceita. Às vezes a família até diz que aceita, mas como é que nós psicólogos percebemos que ela não aceita? Através das orientações que a família se recusa a fazer por acreditar que a criança não precisa, por achar que é difícil. Então existe todo esse entrave [...]. (psicóloga A, 2002).

[...] Às vezes a família não leva essas orientações e não estimula a criança em casa, não porque não queira, mas porque a situação é muito precária e não tem esses estímulos em casa, assim a evolução dessa criança é menor do que o esperado. Às vezes aquela criança que tem até um nível mais leve que outra dentro do grupo, mas como a família não dispõe dos recursos fica mais difícil de fazer essa estimulação [...]. (Psicóloga B, 2020).

As dificuldades no processo de reabilitação são diversas, partindo do início da avaliação neuropsicológica aos processos de reabilitações, porém os maiores entraves que o psicólogo encontra no processo de reabilitação é o contexto familiar, que vária de paciente para paciente, no qual vai variar a relação financeira e até mesmo intelectual da família na qual a crianças está inserida, e Haase (2016) diz que uma das maiores dificuldades na reabilitação autista é a falta do interesse da família na hora de desenvolver e estimular o funcionamento cognitivo e comportamental da criança.

\section{Considerações Finais}

Diante do exposto, o presente estudo tem como finalidade esclarecer ao leitor sobre a atuação do neuropsicólogo, desde a avaliação até o processo em que são escolhidos os métodos de intervenções.

Esses profissionais atuam com a colaboração da família, que são responsáveis pela execução de técnicas no dia a dia e na construção da rotina, bem como auxiliam uma equipe multidisciplinar ou interdisciplinar no cuidado dessas crianças.

A psicoeducação realizada instrui os pais ou responsáveis sobre o TEA além de, melhorar o entendimento sobre as 
dificuldades da criança e como lidar com suas demandas, bem como os possíveis cuidados, e intervenções necessárias para o estímulo do desenvolvimento.

Todo o processo de reabilitação contribui para o desenvolvimento da criança, ajudando nos setores mais afetados, por exemplo, a comunicação, seus relacionamentos interpessoais, seu contexto escolar e meio social. Além de contribuir no investimento das habilidades adquiridas. Portanto, o diagnóstico e a avaliação realizada de forma precoce irão ajudar a garantir um desenvolvimento melhor e mais saudável para essa criança com TEA.

A partir da observação e conhecimentos adquiridos através da pesquisa realizada, é notório que a mesma fornecerá uma contribuição para a sociedade, aonde é ressaltado sobre o diagnóstico, como é a atuação inicial e as intervenções realizadas pelos profissionais, assim como a importância de um acompanhamento especializado para a criança com TEA, além de, ter agregado conhecimentos para os pesquisadores.

\section{Referências}

Almeida, M. A., Boueri, I. Z., \& Postalli, L. M. M. (2016). A Escala de Intensidade de Suporte-SIS no Brasil. Journal of Research in Special Educational Needs, 16, 60-64.

American Psychiatric Association. (2014). DSM-5: Manual diagnóstico e estatístico de transtornos mentais. Artmed Editora.

Bagnasco, A., Tubino, B., Piccotti, E., Rosa, F., Aleo, G., Di Pietro, P., Sasso, L., Gambino, L., Passalacqua, D., \& Emergency and Urgency Department of the IRCCS Giannina Gaslini (2013). Identifying and correcting communication failures among health professionals working in the Emergency Department. International emergency nursing, 21(3), 168-172.

Batista, E. C., Matos, L. A. L., \& Nascimento, A. B. (2017). A entrevista como técnica de investigação na pesquisa qualitativa. Revista Interdisciplinar Científica Aplicada, 11(3), 23-38.

Bardin, L. (2011). Analise de Conteúdo. Ed. Revista Ampliada.

Benitez, P., de Albuquerque, I., Manoni, N. V., Sanches, A. F. R., \& Bondioli, R. (2020). Centro de aprendizagem e desenvolvimento: Estudo de caso interdisciplinar em ABA. Revista Psicologia-Teoria e Prática, 22(1).

Blakleitz, J. S., Paris, T. H., \& Souza, B. (2017). A avalição neuropsicológica no diagnóstico do autismo: uma revisão de literatura. Anais da Jornada Científica dos Campos Gerais, 15.

Camargo, S. P. H., Silva, G. L. D., Crespo, R. O., Oliveira, C. R. D., \& Magalhães, S. L. (2020). Desafios no processo de escolarização de crianças com autismo no contexto inclusivo: diretrizes para formação continuada na perspectiva dos professores. Educação em Revista, 36 .

Fialho. J. (2015). Autismo: A Importância da Intervenção Multidisciplinar. <https://www.comportese.com/2015/06/autismo-a-importancia-da-intervencaomultidisciplinar />

Fuentes, D., Malloy-Diniz, L. F., de Camargo, C. H. P., \& Cosenza, R. M. (2014). Neuropsicologia-: Teoria e Prática. Artmed Editora.

Griesi-Oliveira, K., \& Sertié, A. L. (2017). Transtornos do espectro autista: um guia atualizado para aconselhamento genético. Einstein (São Paulo), 15(2), 233-238.

Haase, V. G., Oliveira, L. D. F. S., Pinheiro, M. I. S., Andrade, P. M. O., de Oliveira Ferreira, F., de Freitas, P. M., \& Teodoro, M. L. M. (2016). Como a neuropsicologia pode contribuir para a educação de pessoas com deficiência intelectual e/ou autismo. Pedagogia em Ação, Belo Horizonte, 8(2).

Ministério da Saúde. (2013) Diretrizes de atenção à reabilitação da pessoa com transtorno do espectro autista. Brasília: Secretaria de Atenção à Saúde, 5-74. https://bvsms.saude.gov.br/bvs/publicacoes/diretrizes_atencao_reabilitacao_pessoa_autismo.pdf

Mozzato, A. R., \& Grzybovski, D. (2011). Análise de conteúdo como técnica de análise de dados qualitativos no campo da administração: potencial e desafios. Revista de Administração Contemporânea, 15(4), 731-747.

Nascimento, S. E. (2020). A Percepção Da Equipe Multiprofissional Em Saúde Na Assistência A Crianças Portadoras De Tea-Transtorno Do Espectro Autista. Revista Científica UMC, 5(3).

Neumann, D. M. C., Tariga, A. R., Perez, D. F., Gomes, P. M., Silveira, J. S., \& Azambuja, L. S. (2017). Avaliação neuropsicológica do transtorno do Espectro Autista. Psicologia. pt

Patias, N. D., \& Von Hohendorff, J. (2019). Critérios de qualidade para artigos de pesquisa qualitativa. Psicologia em estudo, 24.

Pereira, P. L. S., Quintela, E. H. S. X., Chiamulera, T. M., David, A. K. F., Souza, G. A., de Medeiros, P. K. F., \& de Lucena Marcolino, A. B. (2021). Importância da implantação de questionários para rastreamento e diagnóstico precoce do transtorno do espectro autista (TEA) na atenção primária. Brazilian Journal of Health Review, 4(2), 8364-8377. 
Research, Society and Development, v. 10, n. 8, e17010817047, 2021

(CC BY 4.0) | ISSN 2525-3409 | DOI: http://dx.doi.org/10.33448/rsd-v10i8.17047

Setúbal, J. L. (2018). Terapia ABA: conheça esse método para crianças com autismo. <https://institutopensi.org.br/blog-saude-infantil/terapia-abatratamento-autismo/amp/>

Silva, B. G. S., \& Ruiz, E. M. Dificuldades no atendimento multiprofissional em saúde de pessoas com autismo. Direito e cidadania, 53.

Silva, M., \& Mulick, J.A. (2009). Diagnosticando o transtorno autista: aspectos fundamentais e considerações práticas. Psicol. Cienc. Prof, 29(1), 116-121.

Silva, M. R., Araújo, T. C. S., \& Maia, E. M. C. (2018). Aplicação da Psicoeducação na Saúde: revisão integrativa. Revista Brasileira de Psicoterapia, 20(2), 53-63.

Vilar. A. C. Z. L. (2019) Neuropsicologia: uma avaliação atual. <https://rsaude.com.br/campina-grande/materia-destaque/neuropsicologia-uma-avaliacaoatual/18534/>

Yin, R. K. (2016). Pesquisa qualitativa do início ao fim. Métodos de coleta de dados (cap. 6, pp. 138-161). Penso.

Zanchett, S., \& Dallacosta, F. M. (2015). Percepção do profissional da saúde sobre a importância do trabalho multiprofissional e interdisciplinar na atenção básica. Revista Interdisciplinar de Estudos em Saúde, 145-153. 